\title{
Polystyrene Microbeads by Dispersion Polymerization: Effect of Solvent on Particle Morphology
}

\section{Lei Jinhua and Zhou Guangyuan}

Key Laboratory of Polymer Ecomaterials, Changchun Institute of Applied Chemistry, Chinese Academy of Sciences, Changchun 130022, China

Correspondence should be addressed to Zhou Guangyuan; gyzhou1972@163.com

Received 11 November 2013; Revised 21 December 2013; Accepted 21 December 2013; Published 9 January 2014

Academic Editor: Haojun Liang

Copyright ( 2014 L. Jinhua and Z. Guangyuan. This is an open access article distributed under the Creative Commons Attribution License, which permits unrestricted use, distribution, and reproduction in any medium, provided the original work is properly cited.

\begin{abstract}
Polystyrene microspheres (PS) were synthesized by dispersion polymerization in ethanol/2-Methoxyethanol (EtOH/EGME) blend solvent using styrene (St) as monomer, azobisisobutyronitrile (AIBN) as initiator, and PVP (polyvinylpyrrolidone) K-30 as stabilizer. The typical recipe of dispersion polymerization is as follows: St/Solvent/AIBN/PVP $=10 \mathrm{~g} / 88 \mathrm{~g} / 0.1 \mathrm{~g} / 2 \mathrm{~g}$. The morphology of polystyrene microspheres was characterized by the scanning electron microscopy (SEM) and the molecular weights of PS particles were measured by the Ubbelohde viscometer method. The effect of ethanol content in the blend solvent on the morphology and molecular weight of polystyrene was studied. We found that the size of polystyrene microspheres increased and the molecular weight of polystyrene microspheres decreased with the decreasing of the ethanol content in the blend solvent from $100 \mathrm{wt} \%$ to $0 \mathrm{wt} \%$. What is more, the size monodispersity of polystyrene microspheres was quite good when the pure ethanol or pure 2-Methoxyethanol was used; however when the blend ethanol/2-Methoxyethanol solvent was used, the polystyrene microspheres became polydisperse. We further found that the monodispersity of polystyrene microspheres can be significantly improved by adding a small amount of water into the blend solvent; the particles became monodisperse when the content of water in the blend solvent was up to $2 \mathrm{wt} \%$.
\end{abstract}

\section{Introduction}

Micrometer-size monodisperse polymer particles have a wide variety of scientific and technological applications, such as standard calibration, biomedical and clinical diagnosis, highperformance liquid chromatography (HPLC) fillers, catalyst carriers, coatings and ink additives, information storage materials, and colloidal crystals $[1,2]$. Micron-size monodisperse particles were usually difficult to obtain because this size is in-between the diameter range of particles produced by conventional emulsion polymerization $(0.06-0.7 \mu \mathrm{m})$ in a batch process [3-6] and suspension polymerization (50$1000 \mu \mathrm{m})$ [7-11]. Dispersion polymerization is an attractive method for producing micron-size monodisperse polymer particles in a single batch process. Great progress in this field has been achieved over the past three decades [12-21].

Dispersion polymerization in organic hydrocarbon media was first developed by Barrett [22]. Dispersion polymerization may be defined as a type of precipitation polymerization in which one carries out the polymerization of a monomer in the presence of a suitable polymeric stabilizer soluble in the reaction medium. The solvent selected as the reaction medium is a good solvent for both the monomer and the steric stabilizer polymers but a nonsolvent for the polymer being formed.

In order to obtain micron-size monodisperse linear polystyrene beads for seeded polymerization we followed the literature method [23], using ethanol/2-Methoxyethanol blend solvent. We found that in a single solvent, either ethanol or 2-Methoxyethanol, monodisperse polystyrene microspheres were obtained. However, in the ethanol/2Methoxyethanol blend solvent system, we did not get monodisperse polystyrene microspheres but got polydisperse polystyrene microspheres. By chance we found that if a small amount of water was added into the ethanol/2Methoxyethanol blend solvent the uniformity of the resulting polystyrene microspheres will be significantly improved. The effect of the water content in the blend solvent on the morphology and molecular weight of the obtained polystyrene microspheres was further studied. 
TABLE 1: The standard recipe for the dispersion polymerization of styrene $^{\mathrm{a}}$.

\begin{tabular}{lc}
\hline Ingredient & Weight $(\mathrm{g})$ \\
\hline Styrene & 10 \\
Polyvinylpyrrolidone & 2 \\
Solvent & 88 \\
Azobis(isobutyronitrile) & 0.1 \\
\hline
\end{tabular}

${ }^{\mathrm{a}} 70^{\circ} \mathrm{C} ; 24 \mathrm{~h} ; 80 \mathrm{r} / \mathrm{min} ; 10 \mathrm{wt} \%$ of monomer concentration based on total weight.

\section{Experimental}

2.1. Reagents. Styrene (analytical reagent; Beijing Reagents Factory) was washed with a $10 \mathrm{wt} \%$ aqueous sodium hydroxide solution and water, dried over anhydrous magnesium sulfate, and distilled in vacuum. Azobis(isobutyronitrile) (AIBN, Beijing Chemical Factory) was recrystallized in methanol before use. Polyvinylpyrrolidone (PVP, Mw $=4.0$ $\times 104 \mathrm{~g} / \mathrm{mol}$, Sigma) was of reagent grade. Ethanol (analytical reagent; Beijing Reagents Factory) and 2-Methoxyethanol (analytical reagent; Beijing Reagents Factory) were distilled before use.

2.2. Synthesis. Generally, dispersion polymerization is carried out in a four-neck flask with a mechanical stirrer, a reflux condenser, a nitrogen inlet, and a thermometer. To simplify the experimental operation and reduce the system operational differences, a single-necked round bottom flask was used as a reactor; after feeding, purging with nitrogen, the flask was sealed and was placed into an electrical thermostatic oscillation tank and then polymerization started. The polymerization was carried out at $70^{\circ} \mathrm{C}$ oscillation frequency was $80 \mathrm{r} / \mathrm{min}$. The greatest advantage of doing so is a series of comparative experiments which can be carried out simultaneously in the electrical thermostatic oscillation tank. After 24 hours the reaction was terminated. The resulting particles were washed repeatedly using water and ethanol with a centrifuge. Polystyrene microspheres were obtained after drying under vacuum at $60^{\circ} \mathrm{C}$ for $8 \mathrm{~h}$. All ingredients used are summarized in Table 1.

2.3. Measurements. The molecular weights of the polystyrene were tested by Ubbelohde viscometer at $25^{\circ} \mathrm{C}$ using toluene as solvent. Viscosity average molecular weight is calculated by the Mark-Houwink-Sakurada equation: $[\eta]=K M_{v}^{\alpha}(K=$ $0.0075 \mathrm{~mL} / \mathrm{g}, \alpha=0.75)$, where $[\eta]$ is the intrinsic viscosity determined with the Ubbelohde viscometer. The morphology of the particles was characterized using a scanning electron microscope (SEM, XL30ESEM-FEG FEI, USA).

\section{Results and Discussion}

So far there is no uniform polymerization mechanism for dispersion polymerization; currently there are two main explanations: (1) oligomer precipitation mechanism; (2) graft copolymer coalescence mechanism. In this paper the first theory was mainly used to explain the results of the polymerization. Oligomer precipitation mechanism believes that the oligomer formed in early reaction can be dissolved in polymerization solvent. Oligomers precipitate from the solvent when it reached a critical degree of polymerization and nucleus of particle is formed. The nucleus can be present in solvent stably because of the presence of the dispersion stabilizer, and finally the nucleus grows into microspheres.

\subsection{Effect of Ethanol Content on the Polystyrene Microspheres.} Polystyrene microspheres were synthesized by dispersion polymerization in ethanol/2-Methoxyethanol (EtOH/ EGME) blend solvent, and the molecular weights and particle sizes of the polystyrene were controlled by adjusting the ethanol content in the blend solvent. Five comparative experiments with different contents of ethanol are summarized in Table 2 .

From Table 2 we can clearly see that the molecular weights of the polystyrene microspheres became lower with the decreasing of ethanol content in the solvent. This result can be ascribed to the better solubility of styrene in 2Methoxyethanol than that in ethanol; time of chain growth in solvent became longer with the decreasing of ethanol content in the solvent. However, the speed of chain growth in solvent is slower than that of "bulk polymerization" in styrene; finally the lower molecular weight polystyrene was obtained.

From Figure 1 we can clearly see that the particle size of polystyrene microspheres became bigger with the decreasing of the ethanol content in the solvent. This can be ascribed to the better solubility of polystyrene in 2-Methoxyethanol than that in ethanol; the polystyrene chain is looser in 2Methoxyethanol than in ethanol.

From the SEM images of polystyrene microspheres we also found that in a single solvent, either ethanol or 2Methoxyethanol, monodisperse polystyrene microspheres were obtained. However, quite different from those reported in literature [23], in the ethanol/2-Methoxyethanol blend solvent system, we did not get monodisperse polystyrene microspheres but got polydisperse polystyrene microspheres.

3.2. Effect of Water in Blend Solvent on the Polystyrene Microspheres. After comparison with the literature [23] we found the differences. We purified the solvent before use, while without purification in the literature. Small amount of water in solvent may be removed during the solvent refining process. In order to verify this idea, we designed a series of comparative tests; different contents of water were added into the polymerization system (based on PS03). Five comparative experiments with different contents of water are summarized in Table 3.

From Table 3 and Figure 2 we can clearly see that the uniformity of the resulting polystyrene microspheres was significantly improved with the increasing of water content in the solvent. The particles became monodisperse when the content of water in the blend solvent was up to $2 \mathrm{wt} \%$. We believe that this result is associated with the miscibility between solvents. The miscibility between ethanol and 2Methoxyethanol is not so good, while the miscibility between 

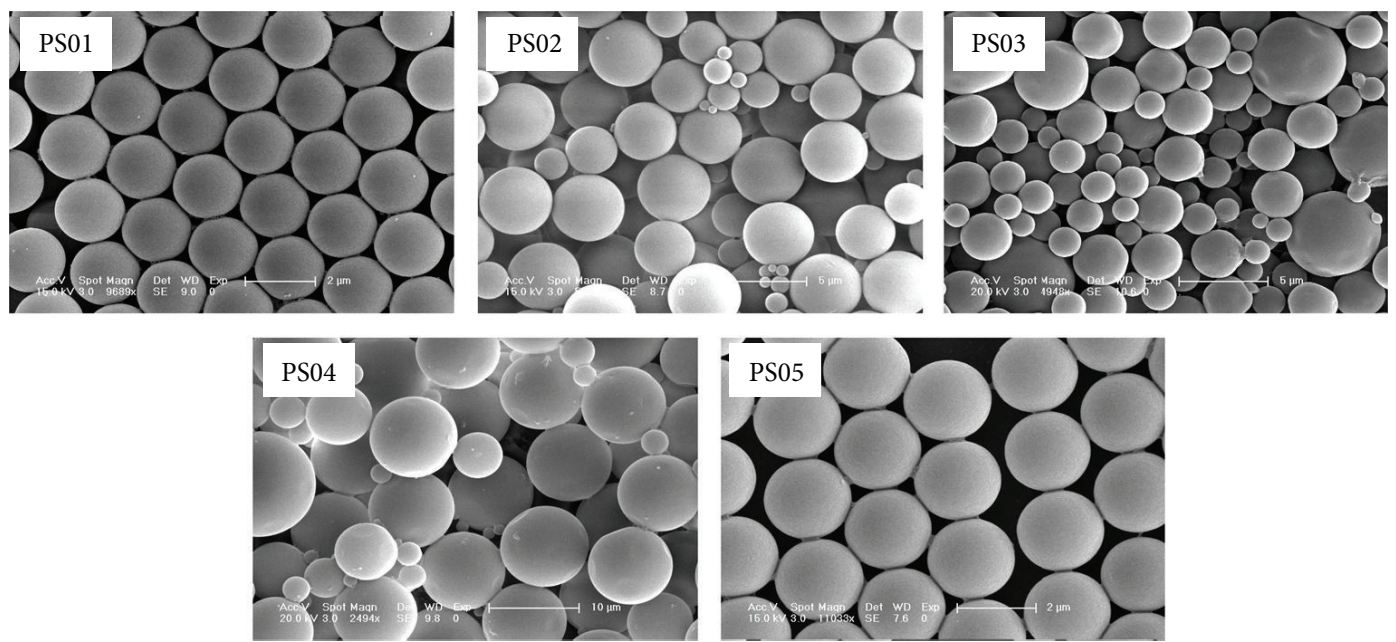

FIGURE 1: SEM images of polystyrene microspheres synthesized with different dosages of ethanol.
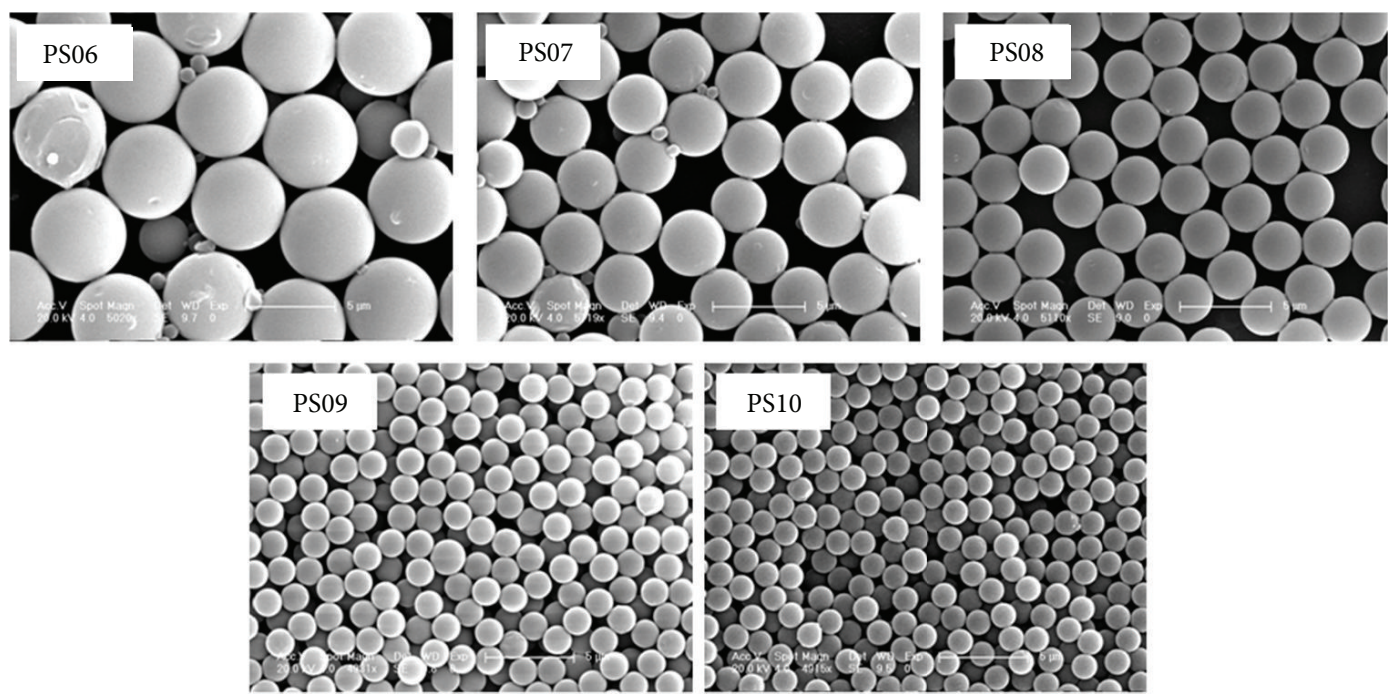

Figure 2: SEM images of polystyrene microspheres synthesized with different dosages of water.

water and ethanol or 2-Methoxyethanol is quite good. Small amount of water in the solvent acted as a "dissolution bridge" between ethanol and 2-Methoxyethanol.

What is more, with the increasing of water content in the solvent, the particle sizes became smaller and the molecular weights of the polystyrene microspheres became higher. This result can be ascribed to the poorer solubility of styrene and polystyrene in water than in ethanol or 2-Methoxyethanol. Water is a poor solvent of styrene.

\section{Conclusion}

Polystyrene microspheres were synthesized by dispersion polymerization in ethanol/2-Methoxyethanol blend solvent. Because the miscibility between ethanol and 2Methoxyethanol is not so good, secondary particles were found and polydisperse polystyrene microspheres were obtained. This problem can be significantly improved when a small amount of water was added into the polymerization system. The particles became monodisperse when the content of water in the blend solvent was up to $2 \mathrm{wt} \%$. This result can be ascribed to the relatively better miscibility between water and ethanol or 2-Methoxyethanol. Small amount of water in the solvent acted as a "dissolution bridge" between ethanol and 2-Methoxyethanol. This result further showed that the solubility between blend solvent should to be attention in the dispersion polymerization in a blend solvent.

\section{Conflict of Interests}

The authors declare that there is no conflict of interests regarding the publication of this paper. 
TABLE 2: Effect of ethanol content on the polystyrene microspheres.

\begin{tabular}{|c|c|c|c|c|c|}
\hline Sample & $\mathrm{EtOH} / \mathrm{g}$ & EGME/g & $M_{v} / 10^{4} \mathrm{~g} \cdot \mathrm{mol}^{-1}$ & $D / \mu \mathrm{m}$ & Morphology \\
\hline PS01 & 88 & 0 & 6.0 & 2 & Monodisperse \\
\hline PS02 & 66 & 22 & 4.9 & $0.3 \sim 4$ & Polydisperse \\
\hline PS03 & 44 & 44 & 3.8 & $0.5 \sim 5$ & Polydisperse \\
\hline PS04 & 22 & 66 & 2.7 & $0.5 \sim 10$ & Polydisperse \\
\hline PS05 & 0 & 88 & 2.0 & 2.2 & Monodisperse \\
\hline
\end{tabular}

TABLE 3: Effect of water in blend solvent on the polystyrene microspheres.

\begin{tabular}{lcccc}
\hline Sample & $\mathrm{H}_{2} \mathrm{O} / \mathrm{wt} \%$ & $M_{v} / 10^{4} \mathrm{~g} \cdot \mathrm{mol}^{-1}$ & $D / \mu \mathrm{m}$ & Morphology \\
\hline PS06 & 0 & 3.7 & $0.5 \sim 5$ & Polydisperse \\
PS07 & 1 & 5.9 & $0.5 \sim 3.5$ & Polydisperse \\
PS08 & 2 & 7.1 & 2.7 & Monodisperse \\
PS09 & 4 & 8.6 & 1.6 & Monodisperse \\
PS10 & 8 & 12 & 1.4 & Monodisperse \\
\hline
\end{tabular}

\section{Acknowledgment}

This work was supported by the National Natural Science Foundation of China for Projects no. 21104073 and no. 51373163.

\section{References}

[1] S. Kawaguchi and K. Ito, "Dispersion polymerization," in Polymer Particles, M. Okubo, Ed., pp. 299-328, Springer, Berlin, Germany, 2005.

[2] J. Ugelstad, A. Berge, T. Ellingsen et al., "Preparation and application of new monosized polymer particles," Progress in Polymer Science, vol. 17, no. 1, pp. 87-161, 1992.

[3] C. S. Chern, "Emulsion polymerization mechanisms and kinetics," Progress in Polymer Science, vol. 31, no. 5, pp. 443-486, 2006.

[4] J. M. Asua, "Emulsion polymerization: from fundamental mechanisms to process developments," Journal of Polymer Science A, vol. 42, no. 5, pp. 1025-1041, 2004.

[5] Q. Wang, S. K. Fu, and T. Y. Yu, "Emulsion polymerization," Progress in Polymer Science, vol. 19, no. 4, pp. 703-753, 1994.

[6] A. Guyot and K. Tauer, "Reactive surfactants in emulsion polymerization," Advances in Polymer Science, vol. 111, pp. 4265, 1994.

[7] B. W. Brooks, "Suspension polymerization processes," Chemical Engineering and Technology, vol. 33, no. 11, pp. 1737-1744, 2010.

[8] F. Machado, E. L. Lima, and J. C. Pinto, "A review on suspension polymerization processes," Polimeros-Ciencia e Tecnologia, vol. 17, no. 2, pp. 166-179, 2007.

[9] P. J. Dowding and B. Vincent, "Suspension polymerisation to form polymer beads," Colloids and Surfaces A, vol. 161, no. 2, pp. 259-269, 2000.

[10] E. Vivaldo-Lima, P. E. Wood, A. E. Hamielec, and A. Penlidis, "An updated review on suspension polymerization," Industrial and Engineering Chemistry Research, vol. 36, no. 4, pp. 939-965, 1997.

[11] H. G. Yuan, G. Kalfas, and W. H. Ray, "Suspension polymerization," Journal of Macromolecular Science, vol. 31, no. 2-3, pp. 215-299, 1991.
[12] W.-D. He, X.-L. Sun, W.-M. Wan, and C.-Y. Pan, "Multiple morphologies of PAA-b-PSt assemblies throughout RAFT dispersion polymerization of styrene with PAA Macro-CTA," Macromolecules, vol. 44, no. 9, pp. 3358-3365, 2011.

[13] J. M. Desimone, E. E. Maury, Y. Z. Menceloglu, J. B. McClain, T. J. Romack, and J. R. Combes, "Dispersion polymerizations in supercritical carbon dioxide," Science, vol. 265, no. 5170, pp. 356-359, 1994.

[14] Y. T. Li and S. P. Armes, "RAFT synthesis of sterically stabilized methacrylic nanolatexes and vesicles by aqueous dispersion polymerization," Angewandte Chemie, vol. 49, no. 24, pp. 40424046, 2010.

[15] W. Q. Shen, Y. Chang, G. Liu, H. Wang, A. Cao, and Z. An, "Biocompatible, antifouling, and thermosensitive coreshell nanogels synthesized by RAFT aqueous dispersion polymerization," Macromolecules, vol. 44, no. 8, pp. 2524-2530, 2011.

[16] S. Sugihara, A. Blanazs, S. P. Armes, A. J. Ryan, and A. L. Lewis, "Aqueous dispersion polymerization: a new paradigm for in situ block copolymer self-assembly in concentrated solution," Journal of the American Chemical Society, vol. 133, no. 39, pp. 15707-15713, 2011.

[17] J. Y. Yuan and M. Antonietti, "Poly(ionic liquid) latexes prepared by dispersion polymerization of ionic liquid monomers," Macromolecules, vol. 44, no. 4, pp. 744-750, 2011.

[18] K. P. Lok and C. K. Ober, "Particle-size control in dispersion polymerization of polystyrene," Canadian Journal of Chemistry, vol. 63, no. 1, pp. 209-216, 1985.

[19] A. J. Paine, "Dispersion polymerization of styrene in polar solvents. 7. A simple mechanistic model to predict particle size," Macromolecules, vol. 23, no. 12, pp. 3109-3117, 1990.

[20] A. J. Paine, W. Luymes, and J. McNulty, "Dispersion polymerization of styrene in polar Solvents. 6. Influence of reaction parameters on particle size and molecular weight in poly $(\mathrm{N}$ vinylpyrrolidone)-stabilized reactions," Macromolecules, vol. 23, no. 12, pp. 3104-3109, 1990.

[21] C. M. Tseng, Y. Y. Lu, M. S. El-Aasser, and J. W. Vanderhoff, "Uniform polymer particles by dispersion polymerization in alcohol," Journal of Polymer Science A, vol. 24, no. 11, pp. 29953007, 1986.

[22] K. E. J. Barrett, Dispersion Polymerization in Organic Media, John Wiley \& Sons, London, UK, 1975.

[23] A. Tuncel, M. Tuncel, and B. Salih, "Electron microscopic observation of uniform macroporous particles. I. Effect of seed latex type and diluent," Journal of Applied Polymer Science, vol. 71, no. 14, pp. 2271-2290, 1999. 

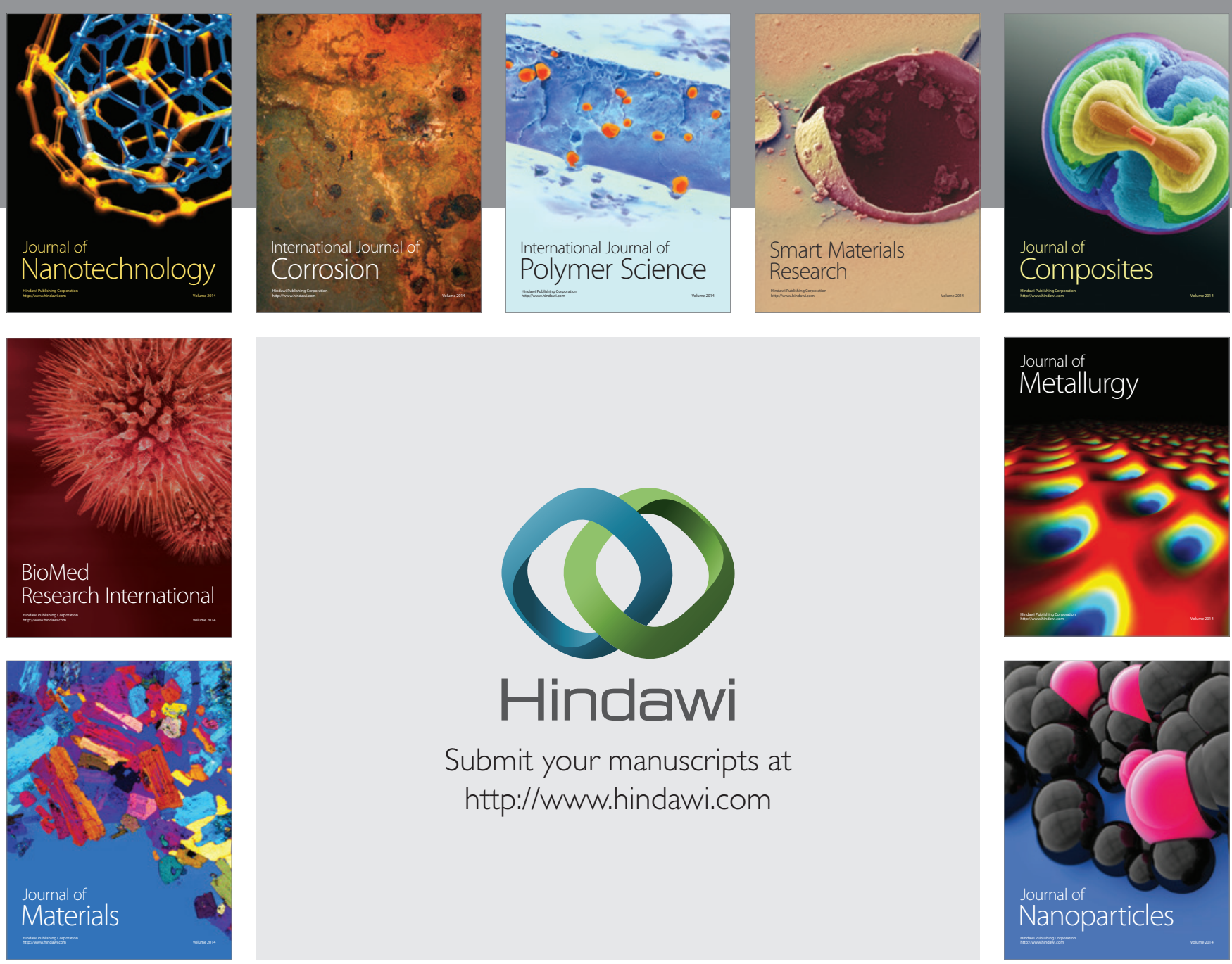

Submit your manuscripts at http://www.hindawi.com
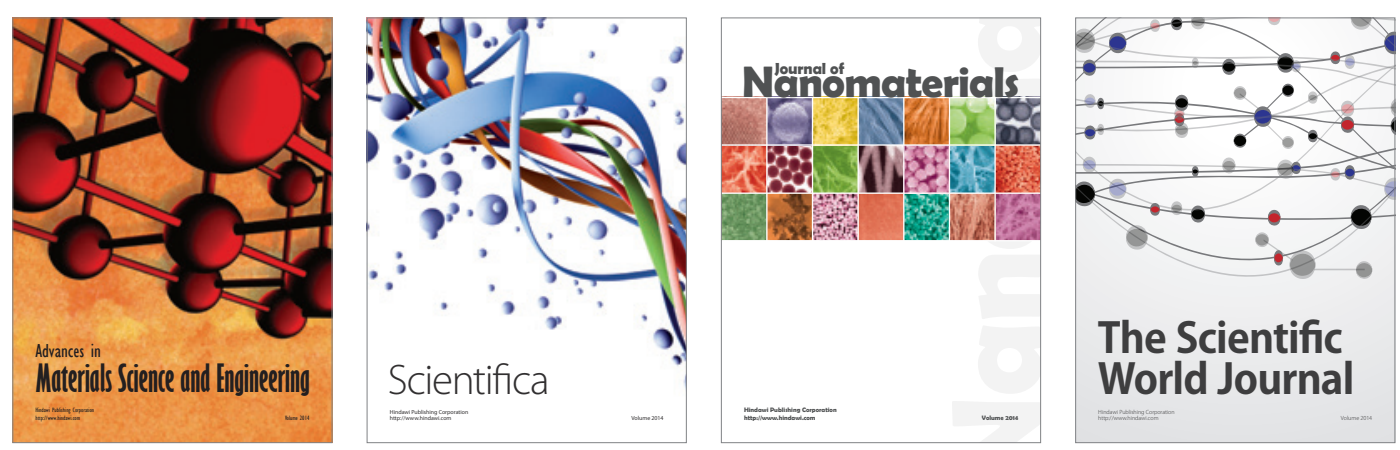

\section{The Scientific World Journal}
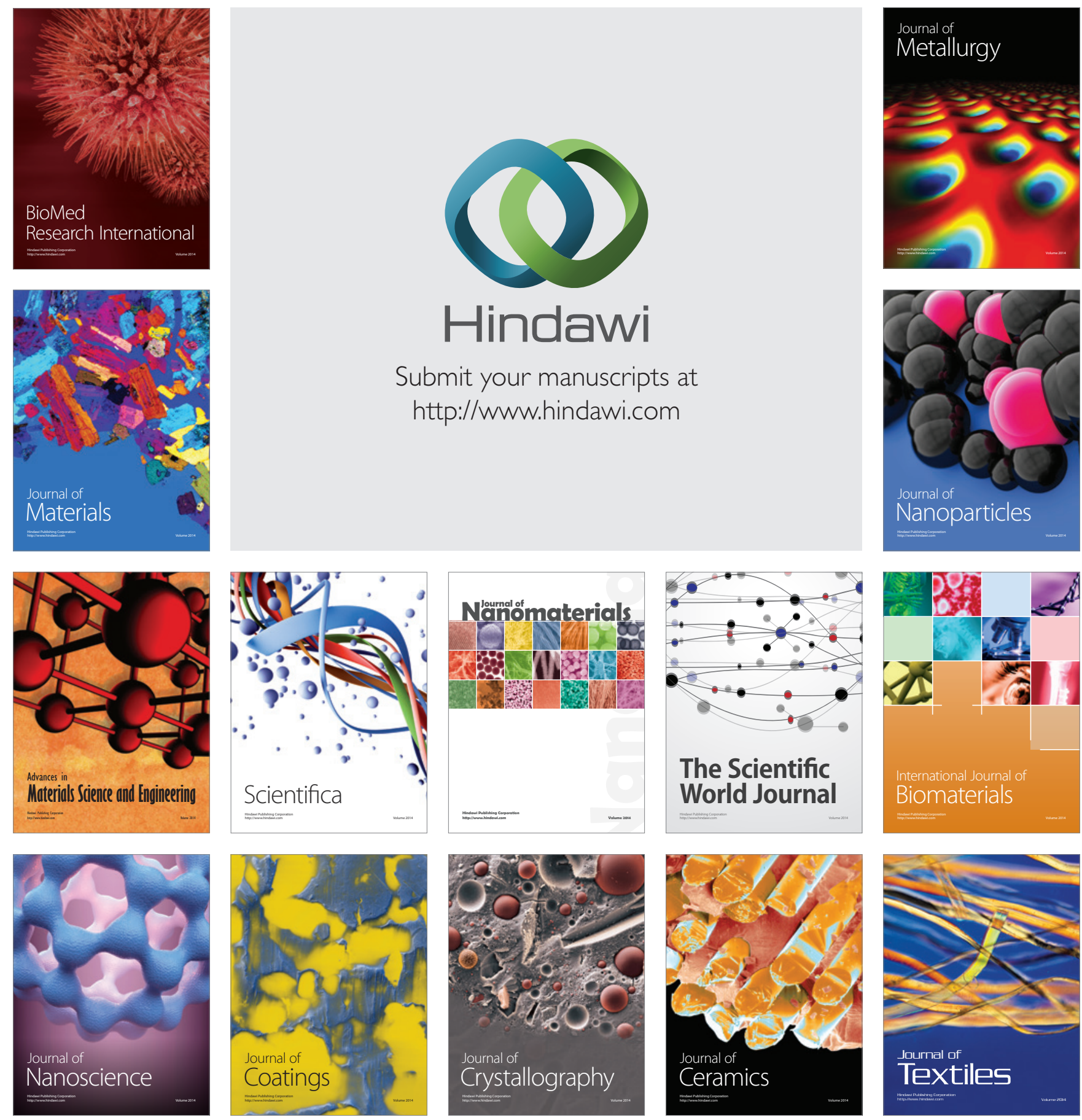